\title{
Existence and uniqueness for p-Laplace equations involving singular nonlinearities
}

Annamaria Canino, Berardino Sciunzi and Alessandro Trombetta

\begin{abstract}
We consider quasilinear elliptic equations involving the $p$-Laplacian and singular nonlinearities. We prove comparison principles and we deduce some uniqueness results.
\end{abstract}

Mathematics Subject Classification. 35J70, 35J92, 35B51.

Keywords. Existence of solutions, Uniqueness of solutions, $p$-Laplace equations.

\section{Contents}

1. Introduction and statement of the results 1

2. Uniqueness of the solution in star-shaped domains 4

3. Uniqueness of the solution in general domains $\quad 7$

4. Appendix: Existence of the solution 14

$\begin{array}{ll}\text { References } & 17\end{array}$

\section{Introduction and statement of the results}

We study uniqueness (and existence) of weak solutions for the following singular quasilinear elliptic problem:

$$
\left\{\begin{array}{cl}
-\Delta_{p} u=\frac{f(x)}{u^{\gamma}} & \text { in } \Omega, \\
u>0 & \text { in } \Omega, \\
u=0 & \text { on } \partial \Omega,
\end{array}\right.
$$

\footnotetext{
BS was partially supported by ERC-2011-Grant: Elliptic PDE's and symmetry of interfaces and layers for odd nonlinearities.

BS was partially supported by PRIN-2011: Variational and Topological Methods in the Study of Nonlinear Phenomena.

AC was partially supported by PRIN-2011: Variational and Topological Methods in the Study of Nonlinear Phenomena.
} 
where $\Omega \subset \mathbb{R}^{N}$ is a bounded smooth domain, $\gamma>0, f \geq 0$ in $\Omega$ (not identically zero) and where $\Delta_{p} u=\operatorname{div}\left(|\nabla u|^{p-2} \nabla u\right)$ is the $p$-Laplace operator, $1<p<$ $+\infty$. Since solutions to $p$-Laplace equations generally are not of class $C^{2}$, a solution to (1.1) has to be understood in the weak distributional meaning. Furthermore, the singular nature of the nonlinearity at zero has to be taken into account. For this reasons, as customary in the literature, we adopt the following definition:

Definition 1.1. We say that $u \in W_{l o c}^{1, p}(\Omega)$ is a weak solution to

$$
-\Delta_{p} u=\frac{f(x)}{u^{\gamma}} \text { in } \Omega
$$

if:

$$
\forall \omega \subset \subset \Omega \exists c_{\omega}: u \geq c_{\omega}>0 \quad \text { in } \omega
$$

and

$$
\int_{\Omega}|\nabla u|^{p-2} \nabla u \nabla \varphi=\int_{\Omega} \frac{f(x) \varphi}{u^{\gamma}} \quad \forall \varphi \in C_{c}^{\infty}(\Omega) .
$$

Generally the solution does not belongs to $W_{0}^{1, p}(\Omega)$. Even in the semilinear case $p=2$, it occurs that solutions are not in $H_{0}^{1}(\Omega)$ for $\gamma \geqslant 3$. This causes that the Dirichlet datum has to be understood in a generalized meaning as here below:

Definition 1.2. We say that $u \leq 0$ on $\partial \Omega$ if $(u-\varepsilon)^{+} \in W_{0}^{1, p}(\Omega)$ for every $\varepsilon>0$. Furthermore $u=0$ on $\partial \Omega$ if $u$ is nonnegative and $u \leq 0$ on $\partial \Omega$.

Starting from the pioneering work [8] many authors studied singular semilinear elliptic equations, see e.g. [4-6,13,15,16,18,19,22]. Let us also mention the contributions in $[1,3,14]$ where related problems involving first order terms are considered.

In this paper we deal with the uniqueness (and existence) issue for the quasilinear problem (1.1). Actually the existence of a solution, under suitable assumptions on $f$, follows relying on the technique introduced in [4] which is based on a truncation argument and on a regularization argument. In [4] the semilinear case $p=2$ is considered but the adaptation of the technique to the quasilinear case is not difficult. Anyway, since some technical changes are needed, we will provide the details of the proofs in the appendix while we state here the result:

Theorem 1.3. For $\gamma \geq 1$ and $p>1$ problem (1.1) has a solution $u \in W_{l o c}^{1, p}(\Omega)$ provided that $f \in L^{1}(\Omega)$. Such a solution fulfills the boundary datum in the sense that $u^{\frac{\gamma+p-1}{p}} \in W_{0}^{1, p}(\Omega)$ and also in the meaning of Definition 1.2.

In the case $0<\gamma<1$ and $1<p<N$ problem (1.1) has a solution $u \in W_{0}^{1, p}(\Omega)$ if $f \in L^{m}(\Omega)$ with

$$
m=\frac{N p}{N(p-1)+p+\gamma(N-p)}=\left(\frac{p^{*}}{1-\gamma}\right)^{\prime} .
$$


If $p=N$ and $0<\gamma<1$ the same result follows assuming that $f \in L^{m}(\Omega)$ for some $m>1$ while in the case $p>N$ and $0<\gamma<1$ the result is true if $f \in L^{1}(\Omega)$.

The main issue considered here is the uniqueness problem.

Though the uniqueness is expected because the nonlinearity is decreasing in $u$, only partial results have been provided in the literature. This is mainly caused by the fact that the solutions generally are not in $W_{0}^{1, p}(\Omega)$. On the other hand, it is not difficult to prove a uniqueness result via standard arguments in $W_{0}^{1, p}(\Omega)$. This is the case also when restricting the attention to solutions that are continuous up to the boundary. We refer the readers e.g. to $[5,8]$. Since in our case solutions are not in general in $W_{0}^{1, p}(\Omega)$ and generally are not continuous up to the boundary a positive answer to the uniqueness of the solutions is still not known. Recently a positive answer has been provided in [7] under very mild assumptions on $f$ and in the semilinear case $p=2$. The quasilinear case is still undertaken and, unfortunately, the technique exploited in [7] is very much related to the linear nature of the Laplace operator and therefore it is difficult to exploit it in the quasilinear setting.

We provide here two different results based on two new different techniques. The first result holds in star-shaped domains.

Theorem 1.4. Let $\gamma>0, f \in L^{1}(\Omega)$. Then, if $\Omega$ is star-shaped with respect to the origin, the solution to problem (1.1) is unique.

More precisely, if $u$ and $v$ are solutions to (1.1) that fulfill the Dirichlet datum in the sense of Definition 1.2 then it follows that $u=v$. The same holds if $u$ and $v$ are solutions to (1.1) such that $u^{\frac{\gamma+p-1}{p}} \in W_{0}^{1, p}(\Omega)$ and $v^{\frac{\gamma+p-1}{p}} \in$ $W_{0}^{1, p}(\Omega)$.

The proof of Theorem 1.4 is based on a scaling argument and on a fine asymptotic analysis. The lack of regularity of the solutions up to the boundary causes that standard test functions techniques cannot be exploited and the star-shaped geometry of the domains is needed to apply the scaling argument. We can avoid the restriction regarding the geometry of the domain if we require some further summability assumptions. Namely we have the following:

Theorem 1.5. Let us consider problem (1.1) and assume that $f \in L^{1}(\Omega)$ for $0<\gamma \leq 1$ while, in the case $\gamma>1$, we assume that $f \in L^{m}(\Omega)$ with $m>\frac{N}{p}$ if $1<p<N, f \in L^{m}(\Omega)$ with $m>1$ if $p=N, f \in L^{1}(\Omega)$ if $p>N$.

Then the solution is unique.

In the whole paper the reader will guess that the case $\gamma \geq 1$ is the most difficult one to be considered. In the case $0<\gamma<1$, according to Theorem 1.3, we consider solutions in $W_{0}^{1, p}(\Omega)$. The existence of such solutions is proved in Theorem 1.3 requiring extra summability assumptions on $f$. Such restriction is anyway not needed when dealing with the problem of uniqueness. Furthermore a solution that fulfills the boundary datum in the sense that $u^{\frac{\gamma+p-1}{p}} \in W_{0}^{1, p}(\Omega)$, also fulfills the boundary datum in the meaning of Definition 1.2. This follows 
by direct computation exploiting the fact that $u$ is strictly positive in the support of $(u-\varepsilon)^{+}$.

Theorem 1.5 is proved via a comparison argument based on a suitable regularized problem. Namely standard techniques are applied to a suitable regularized problem and the comparison principle follows passing to the limit. The limiting process in this case is not standard because of the presence of the singular nonlinearity in the right hand side.

The paper is organized as follows. In Sect. 2 we prove the uniqueness result in star-shaped domains, namely we prove Theorem 1.4. In Sect. 3 we prove the uniqueness of the solution in general domains, namely we prove Theorem 1.5. We postpone the proof of the existence result, namely Theorem 1.3 , in the appendix.

\section{Uniqueness of the solution in star-shaped domains}

In this section we will prove Theorem 1.4. The proof works in this case in the same way for any $\gamma>0$, anyway the result is crucial in the case $\gamma>1$ since here we only assume that $f \in L^{1}(\Omega)$.

In the following we will frequently use the following well known elliptic estimates (see [21]):

Lemma 2.1. $\forall p>1$ there exist positive constants $C_{1}, C_{2}$, depending on $p$, such that $\forall \eta, \eta^{\prime} \in \mathbb{R}^{N}$ with $|\eta|+\left|\eta^{\prime}\right|>0$

$$
\begin{aligned}
{\left[|\eta|^{p-2} \eta-\left|\eta^{\prime}\right|^{p-2} \eta^{\prime}\right]\left[\eta-\eta^{\prime}\right] } & \geq C_{1}\left(|\eta|+\left|\eta^{\prime}\right|\right)^{p-2}\left|\eta-\eta^{\prime}\right|^{2} \\
\left.|| \eta\right|^{p-2} \eta-\left|\eta^{\prime}\right|^{p-2} \eta^{\prime} \mid & \leq C_{2}\left(|\eta|+\left|\eta^{\prime}\right|\right)^{p-2}\left|\eta-\eta^{\prime}\right| .
\end{aligned}
$$

Proof of Theorem 1.4 We will carry out the proof in the case $\gamma>1$. The case $0<\gamma \leq 1$ is simpler and only trivial modifications are needed.

Also we will consider the case of solutions $u, v$ such that $u^{\frac{\gamma+p-1}{p}} \in$ $W_{0}^{1, p}(\Omega)$ and $v^{\frac{\gamma+p-1}{p}} \in W_{0}^{1, p}(\Omega)$. When the Dirichlet datum is understood as in Definition 1.2 only trivial modifications are needed that will be listed below.

Fix $0<\varepsilon<1$ and set

$$
v_{\varepsilon}(x):=\varepsilon^{\frac{p}{\gamma+p-1}} v\left(\frac{x}{\varepsilon}\right) \quad \text { in } \Omega_{\varepsilon}:=\varepsilon \Omega .
$$

In the following we consider $v_{\varepsilon}$ defined in $\Omega$ by setting it equal to zero in $\Omega \backslash \Omega_{\varepsilon}$. It is easy to check that, in the distributional meaning, we have

$$
-\Delta_{p} v_{\varepsilon}=\frac{f_{\varepsilon}}{v_{\varepsilon}^{\gamma}} \quad \text { in } \Omega_{\varepsilon}
$$

where $f_{\varepsilon}(x):=f\left(\frac{x}{\varepsilon}\right)$. For any $\delta>0$ given, we also set

$$
u_{\delta}:=u+\delta \text {, }
$$

so that, in the weak distributional meaning, we have

$$
-\Delta_{p} u_{\delta} \geq \frac{f}{u_{\delta}^{\gamma}} \quad \text { in } \Omega \text {. }
$$


We claim that:

$$
\left(v_{\varepsilon}-u_{\delta}\right)^{+} \in W_{0}^{1, p}\left(\Omega_{\varepsilon}\right) .
$$

To prove this note that, since $v_{\varepsilon}^{\frac{\gamma+p-1}{p}} \in W_{0}^{1, p}\left(\Omega_{\varepsilon}\right)$, then there exists a sequence $\varphi_{n} \in C_{c}^{\infty}\left(\Omega_{\varepsilon}\right), \varphi_{n} \geq 0$, such that

$$
\varphi_{n} \stackrel{W_{0}^{1, p}\left(\Omega_{\varepsilon}\right)}{\rightarrow} v_{\varepsilon}^{\frac{\gamma+p-1}{p}}
$$

Let us set

$$
w_{n}:=\left(\varphi_{n}^{\frac{p}{\gamma+p-1}}-u_{\delta}\right)^{+}
$$

and observe that $\operatorname{supp} w_{n} \subset \subset \Omega_{\varepsilon}$ and

$$
\varphi_{n}>\delta^{\frac{\gamma+p-1}{p}},
$$

in the support of $w_{n}$.

Clearly $\varphi_{n}$ is uniformly bounded in $W_{0}^{1, p}\left(\Omega_{\varepsilon}\right)$ so that

$$
\begin{aligned}
\int_{\operatorname{supp} w_{n}}\left|\nabla\left(\varphi_{n}^{\frac{p}{\gamma+p-1}}\right)\right|^{p} & =\left(\frac{p}{\gamma+p-1}\right)^{p} \int_{\operatorname{supp} w_{n}} \frac{1}{\varphi_{n}^{\frac{(\gamma-1) p}{\gamma+p-1}}}\left|\nabla \varphi_{n}\right|^{p} \\
& <\left(\frac{p}{\gamma+p-1}\right)^{p} \frac{1}{\delta \gamma-1} \int_{\operatorname{supp} w_{n}}\left|\nabla \varphi_{n}\right|^{p} \\
& \leq \text { Const. }
\end{aligned}
$$

Then

$$
\begin{aligned}
\left\|w_{n}\right\|_{W_{0}^{1, p}\left(\Omega_{\varepsilon}\right)}^{p} & =\int_{\Omega_{\varepsilon}}\left|\nabla\left(\varphi_{n}^{\frac{p}{\gamma+p-1}}-u_{\delta}\right)^{+}\right|^{p} \leq \int_{\operatorname{supp} w_{n}}\left|\nabla \varphi_{n}^{\frac{p}{\gamma+p-1}}-\nabla u_{\delta}\right|^{p} \\
& \leq C \int_{\operatorname{supp} w_{n}}\left(\left|\nabla \varphi_{n}^{\frac{p}{\gamma+p-1}}\right|^{p}+\left|\nabla u_{\delta}\right|^{p}\right) \\
& \leq C\left(\int_{\operatorname{supp} w_{n}}\left|\nabla \varphi_{n}^{\frac{p}{\gamma+p-1}}\right|^{p}+\int_{\Omega_{\varepsilon}}|\nabla u|^{p}\right) \leq \text { Const. }
\end{aligned}
$$

It follows that, up to subsequences, $w_{n}$ weakly converges to $\bar{w} \in W_{0}^{1, p}\left(\Omega_{\varepsilon}\right)$ and $w_{n}$ a.e. converges to $\bar{w}$ in $\Omega_{\varepsilon}$. Since we also now that $w_{n}$ a.e. converges to $\left(v_{\varepsilon}-u_{\delta}\right)^{+}$in $\Omega_{\varepsilon}$, then we have $\bar{w}=\left(v_{\varepsilon}-u_{\delta}\right)^{+}$and the claim is proved. Note that the same claim follows when the Dirichlet datum is understood in the meaning of Definition 1.2. The proof in this case is simpler since the support of $\left(v_{\varepsilon}-u_{\delta}\right)^{+}$is contained in the support of $\left(v_{\varepsilon}-\delta\right)^{+}$.

Now we set $T_{k}(s):=\min \{s, k\}, k>0$. We have that

$$
T_{k}\left(\left(v_{\varepsilon}-u_{\delta}\right)^{+}\right) \in W_{0}^{1, p}\left(\Omega_{\varepsilon}\right) \cap L^{\infty}\left(\Omega_{\varepsilon}\right) .
$$

Let $\psi_{n} \in C_{c}^{\infty}\left(\Omega_{\varepsilon}\right), \psi_{n} \geq 0$, such that

$$
\psi_{n} \stackrel{W_{0}^{1, p}\left(\Omega_{\varepsilon}\right)}{\rightarrow}\left(v_{\varepsilon}-u_{\delta}\right)^{+} .
$$

We have that $T_{k}\left(\psi_{n}\right) \in W_{0}^{1, p}\left(\Omega_{\varepsilon}\right) \cap L^{\infty}\left(\Omega_{\varepsilon}\right)$ and

$$
T_{k}\left(\psi_{n}\right) \stackrel{W_{0}^{1, p}\left(\Omega_{\varepsilon}\right)}{\rightarrow} T_{k}\left(\left(v_{\varepsilon}-u_{\delta}\right)^{+}\right) .
$$


Setting

$$
\tilde{T}_{k}\left(\psi_{n}\right):=\min \left\{T_{k}\left(\psi_{n}\right), T_{k}\left(\left(v_{\varepsilon}-u_{\delta}\right)^{+}\right)\right\},
$$

we have

$$
\operatorname{supp} \tilde{T}_{k}\left(\psi_{n}\right) \subset \operatorname{supp} T_{k}\left(\left(v_{\varepsilon}-u_{\delta}\right)^{+}\right) \text {. }
$$

Choosing $\tilde{T}_{k}\left(\psi_{n}\right)$ as test function in (2.2) and (2.3), we get:

$$
\begin{aligned}
& \int_{\Omega_{\varepsilon}}\left(\left|\nabla v_{\varepsilon}\right|^{p-2} \nabla v_{\varepsilon}-\left|\nabla u_{\delta}\right|^{p-2} \nabla u_{\delta}\right) \nabla \tilde{T}_{k}\left(\psi_{n}\right) \\
& \leq \int_{\Omega_{\varepsilon}}\left(\frac{f_{\varepsilon}}{v_{\varepsilon}^{\gamma}}-\frac{f}{u_{\delta}^{\gamma}}\right) \tilde{T}_{k}\left(\psi_{n}\right) \\
& \quad=\int_{\Omega_{\varepsilon}} f_{\varepsilon}\left(\frac{1}{v_{\varepsilon}^{\gamma}}-\frac{1}{u_{\delta}^{\gamma}}\right) \tilde{T}_{k}\left(\psi_{n}\right)+\int_{\Omega_{\varepsilon}} \frac{f_{\varepsilon}-f}{u_{\delta}^{\gamma}} \tilde{T}_{k}\left(\psi_{n}\right) \\
& \leq \int_{\Omega_{\varepsilon}} \frac{f_{\varepsilon}-f}{u_{\delta}^{\gamma}} \tilde{T}_{k}\left(\psi_{n}\right) .
\end{aligned}
$$

Exploiting the Lebesgue Theorem, we pass to the limit and get

$$
C \int_{\Omega_{\varepsilon}}\left(\left|\nabla v_{\varepsilon}\right|+\left|\nabla u_{\delta}\right|\right)^{p-2}\left|\nabla T_{k}\left(\left(v_{\varepsilon}-u_{\delta}\right)^{+}\right)\right|^{2} \leq \int_{\Omega_{\varepsilon}} \frac{f_{\varepsilon}-f}{u_{\delta}^{\gamma}} T_{k}\left(\left(v_{\varepsilon}-u_{\delta}\right)^{+}\right) \text {. }
$$

Now we fix $\sigma>0$ and use the notation $x:=\varepsilon y$. By Luzin's Theorem we can choose a compact $E_{\sigma} \subset \Omega$ with $\mathcal{L}\left(\Omega \backslash E_{\sigma}\right)<\sigma$ (where $\mathcal{L}$ is the Lebesgue measure), such that $u$ and $v$ are continuous in $E_{\sigma}$. It is convenient to replace $E_{\sigma}$ by it's interior in the following computations in order to use the continuity of $u$ and $v$ in an open set. This is possible recalling that the boundary has zero Lebesgue measure. We use the same notation. We get that

$$
\begin{aligned}
\mid \int_{\Omega_{\varepsilon}} & \frac{f_{\varepsilon}}{u_{\delta}^{\gamma}} T_{k}\left(\left(v_{\varepsilon}-u_{\delta}\right)^{+}\right) d x-\int_{\Omega} \frac{f(y)}{u_{\delta}^{\gamma}(y)} T_{k}\left(\left(v(y)-u_{\delta}(y)\right)^{+}\right) d y \mid \\
= & \mid \int_{\Omega} \frac{\varepsilon^{N} f(y)}{u_{\delta}^{\gamma}(\varepsilon y)} T_{k}\left(\left(v_{\varepsilon}(\varepsilon y)-u_{\delta}(\varepsilon y)\right)^{+}\right) d y \\
& -\int_{\Omega} \frac{f(y)}{u_{\delta}^{\gamma}(y)} T_{k}\left(\left(v(y)-u_{\delta}(y)\right)^{+}\right) d y \mid \\
\leq & \mid \int_{E_{\sigma}}\left[\frac{\varepsilon^{N} f(y)}{u_{\delta}^{\gamma}(\varepsilon y)} T_{k}\left(\left(v_{\varepsilon}(\varepsilon y)-u_{\delta}(\varepsilon y)\right)^{+}\right)\right. \\
& \left.-\frac{f(y)}{u_{\delta}^{\gamma}(y)} T_{k}\left(\left(v(y)-u_{\delta}(y)\right)^{+}\right)\right] d y \mid \\
& +\left|\int_{\Omega \backslash E_{\sigma}} \frac{\varepsilon^{N} f(y)}{u_{\delta}^{\gamma}(\varepsilon y)} T_{k}\left(\left(v_{\varepsilon}(\varepsilon y)-u_{\delta}(\varepsilon y)\right)^{+}\right) d y\right| \\
+ & \left|\int_{\Omega \backslash E_{\sigma}} \frac{f(y)}{u_{\delta}^{\gamma}(y)} T_{k}\left(\left(v(y)-u_{\delta}(y)\right)^{+}\right) d y\right| .
\end{aligned}
$$


We note now that, since $u$ and $v$ are continuous in $E_{\sigma}$, then $u_{\delta}(\varepsilon y)$ converges to $u_{\delta}$ and $v_{\varepsilon}(\varepsilon y)$ converges to $v$ in $E_{\sigma}$, as $\varepsilon \rightarrow 1$. This allows to pass to the limit via the Lebesgue Theorem.

Furthermore, the other terms (namely the integrals outside $E_{\sigma}$ ), are arbitrary small since $\mathcal{L}\left(\Omega \backslash E_{\sigma}\right)$ is arbitrary small and $f \in L^{1}(\Omega)$. From this we easily infer that

$$
\int_{\Omega_{\varepsilon}} \frac{f_{\varepsilon}}{u_{\delta}^{\gamma}} T_{k}\left(\left(v_{\varepsilon}-u_{\delta}\right)^{+}\right) d x \underset{\varepsilon \rightarrow 1}{\longrightarrow} \int_{\Omega} \frac{f}{u_{\delta}^{\gamma}} T_{k}\left(\left(v-u_{\delta}\right)^{+}\right) d x
$$

and, exploiting the fact that $f \in L^{1}(\Omega)$, we have that

$$
\int_{\Omega_{\varepsilon}} \frac{f_{\varepsilon}-f}{u_{\delta}^{\gamma}} T_{k}\left(\left(v_{\varepsilon}-u_{\delta}\right)^{+}\right) \underset{\varepsilon \rightarrow 1}{\longrightarrow} 0 .
$$

By [9] it follows that $\nabla v_{\varepsilon}$ almost everywhere converges to $\nabla v$. This can be deduced by (2.2) exploiting [9] in each subset of the domain, recalling that the $L^{1}$-convergence of the right hand side follows since $v$ is positive in the interior of the domain. Therefore, letting $\varepsilon \rightarrow 1$ in (2.4), we obtain

$$
\int_{\Omega}\left(|\nabla v|+\left|\nabla u_{\delta}\right|\right)^{p-2}\left|\nabla T_{k}\left(\left(v-u_{\delta}\right)^{+}\right)\right|^{2}=0
$$

so that $v \leq u_{\delta}$ in $\Omega$. By the arbitrariness of $\delta$ we have $v \leq u$ in $\Omega$ and the thesis follows reversing the roles of $u$ and $v$.

\section{Uniqueness of the solution in general domains}

In this section we prove Theorem 1.5. We start considering the regularized problem:

$$
\begin{cases}-\Delta_{p} u=f(x) g_{h}(u) & \text { in } \Omega \\ u>0 & \text { in } \Omega \\ u=0 & \text { on } \partial \Omega\end{cases}
$$

where $g_{h}(s)=\min \left\{\frac{1}{s^{\gamma}}, h\right\}, s>0$. We will find a solution to (3.1) looking at the problem:

$$
\begin{cases}-\Delta_{p} u_{n}=f_{n}(x) g_{h}\left(u_{n}\right) & \text { in } \Omega, \\ u_{n}=0 & \text { on } \partial \Omega,\end{cases}
$$

where $f_{n}(x):=\min \{f(x), n\}$.

We have the following

Lemma 3.1. For each $n \in \mathbb{N}$, there exists $u_{n} \in W_{0}^{1, p}(\Omega)$ weak solution to (3.2).

Proof. For each $v \in L^{p}(\Omega)$ there exists a unique $w \in W_{0}^{1, p}(\Omega)$ weakly solving

$$
\left\{\begin{array}{cl}
-\Delta_{p} w=f_{n}(x) g_{h}(|v|) & \text { in } \Omega, \\
w=0 & \text { on } \partial \Omega
\end{array}\right.
$$


To prove this just consider the associated energy functional $J: W_{0}^{1, p}(\Omega) \rightarrow \mathbb{R}$ defined by

$$
\begin{aligned}
J(w) & =\frac{1}{p} \int_{\Omega}|\nabla w|^{p}-\int_{\Omega} f_{n} g_{h}(|v|) w \\
& =\frac{1}{p}\|\nabla w\|_{L^{p}(\Omega)}^{p}-\int_{\Omega} f_{n} g_{h}(|v|) w .
\end{aligned}
$$

It follows easily that $J$ is continuous, strictly convex and coercive so that the existence and the uniqueness of the solution to (3.3) follow by standard arguments.

Therefore we can define the operator:

$$
v \in L^{p}(\Omega) \rightarrow w=S(v) \in L^{p}(\Omega),
$$

where $w$ is the solution to (3.3). It is not difficult to prove via standard arguments that $S$ is continuous and compact. Furthermore, we claim that there exists a radius $R>0$ such that the ball in $L^{p}(\Omega)$ of radius $R$ is invariant under the action of the operator $S$.

To prove the claim, let us plug $w$ as test function in (3.3). Exploiting the fact that $f_{n} \leq n$ and $g_{h}(|v|) \leq h$, we get

$$
\int_{\Omega}|\nabla w|^{p}=\int_{\Omega} f_{n} g_{h}(|v|) w \leq n h \int_{\Omega}|w| \leq n h|\Omega|^{\frac{p-1}{p}}\left(\int_{\Omega}|w|^{p}\right)^{\frac{1}{p}} .
$$

Therefore, by the Poincaré's inequality, we deduce

$$
\int_{\Omega}|w|^{p} \leq C n h|\Omega|^{\frac{p-1}{p}}\left(\int_{\Omega}|w|^{p}\right)^{\frac{1}{p}}
$$

so that

$$
\|w\|_{L^{p}(\Omega)} \leq\left(C n h|\Omega|^{\frac{p-1}{p}}\right)^{\frac{1}{p-1}}:=R
$$

proving the claim.

We can consequently exploit Schauder's Fixed Point Theorem to deduce that there exists $u_{n} \in W_{0}^{1, p}(\Omega)$ such that $u_{n}=S\left(u_{n}\right)$, namely $u_{n}$ is a solution to $(3.2)$.

Remark 3.2. We remark that, since $f_{n} g_{h}\left(\left|u_{n}\right|\right) \geq 0$ in $\Omega$, then the weak maximum principle (see e.g. [21]) implies that $u_{n} \geq 0$ in $\Omega$. Furthermore, by the strong maximum principle (see $[21,27]$ ), we infer that $u_{n}>0$ in $\Omega$ since our problem does not admits the trivial solution. Finally, since $f_{n} g_{h} \in L^{\infty}$, by standard regularity results $[10-12,17,20,23-26]$, it follows that $u_{n} \in C^{1, \alpha}(\bar{\Omega})$ for some $\alpha \in(0,1)$.

Lemma 3.3. Let $u_{n}$ be the solution to (3.2) given by Lemma 3.1. Then $u_{n}$ is unique and the family $\left\{u_{n}\right\}$ is increasing with respect to $n$. Furthermore

$$
\forall \omega \subset \subset \Omega \exists c_{\omega}: \forall n \in \mathbb{N}, u_{n} \geq c_{\omega}>0 \quad \text { in } \omega .
$$


Proof. Since $0 \leq f_{n} \leq f_{n+1}$ we have

$$
\begin{aligned}
\int_{\Omega}\left|\nabla u_{n}\right|^{p-2} \nabla u_{n} \nabla \varphi & =\int_{\Omega} f_{n} g_{h}\left(u_{n}\right) \varphi \leq \int_{\Omega} f_{n+1} g_{h}\left(u_{n}\right) \varphi, \\
\int_{\Omega}\left|\nabla u_{n+1}\right|^{p-2} \nabla u_{n+1} \nabla \varphi & =\int_{\Omega} f_{n+1} g_{h}\left(u_{n+1}\right) \varphi,
\end{aligned}
$$

for each $\varphi \in C_{c}^{\infty}(\Omega)$ with $\varphi \geq 0$ in $\Omega$. By standard density arguments (exploiting the fact that the regularized problem is not singular) we see that $\varphi=\left(u_{n}-u_{n+1}\right)^{+}$can be used as test function. Therefore, exploiting also the fact that $g_{h}$ is non-increasing, we obtain

$$
\begin{aligned}
& \int_{\Omega}\left(\left|\nabla u_{n}\right|+\left|\nabla u_{n+1}\right|\right)^{p-2}\left|\nabla\left(u_{n}-u_{n+1}\right)^{+}\right|^{2} \\
& \quad \leq C \int_{\Omega}\left(\left|\nabla u_{n+1}\right|^{p-2} \nabla u_{n+1}-\left|\nabla u_{n}\right|^{p-2} \nabla u_{n}\right) \nabla\left(u_{n}-u_{n+1}\right)^{+} \leq 0 .
\end{aligned}
$$

which implies $\left(u_{n}-u_{n+1}\right)^{+}=0$ a.e. in $\Omega$ and therefore $u_{n} \leq u_{n+1}$ in $\Omega$.

The fact that $u_{n}$ is strictly bounded away from zero in the interior of $\Omega$ follows now by the fact that $u_{1}$ is strictly positive by the strong maximum principle.

The uniqueness of the solution follows with similar arguments. Let $n \in \mathbb{N}$ and consider two solutions $u_{n}$ and $v_{n}$, so that

$$
\begin{gathered}
\int_{\Omega}\left(\left|\nabla u_{n}\right|^{p-2} \nabla u_{n}-\left|\nabla v_{n}\right|^{p-2} \nabla v_{n}\right) \nabla \varphi \\
=\int_{\Omega} f_{n}\left(g_{h}\left(u_{n}\right)-g_{h}\left(v_{n}\right)\right) \varphi
\end{gathered}
$$

for each $\varphi \in C_{c}^{\infty}(\Omega), \varphi \geq 0$. Choosing $\varphi=\left(u_{n}-v_{n}\right)^{+}$, observing that $g_{h}$ is decreasing, we obtain

$$
\begin{aligned}
& C \int_{\Omega}\left(\left|\nabla u_{n}\right|+\left|\nabla v_{n}\right|\right)^{p-2}\left|\nabla\left(u_{n}-v_{n}\right)^{+}\right|^{2} \\
& \quad \leq \int_{\Omega}\left(\left|\nabla u_{n}\right|^{p-2} \nabla u_{n}-\left|\nabla v_{n}\right|^{p-2} \nabla v_{n}\right) \nabla\left(u_{n}-v_{n}\right)^{+} \\
& \quad \leq \int_{\Omega} f_{n}\left(g_{h}\left(u_{n}\right)-g_{h}\left(v_{n}\right)\right)\left(u_{n}-v_{n}\right)^{+} \leq 0
\end{aligned}
$$

From this we infer that $\left(u_{n}-v_{n}\right)^{+}=0$ a.e. in $\Omega$ and $u_{n} \leq v_{n}$. By symmetry, this also implies $u_{n}=v_{n}$.

Lemma 3.4. Let $1<p<N$ and let $u_{n}$ be the solution to (3.2) and assume that $f \in L^{m}(\Omega)$, with

$$
m=\frac{N p}{N(p-1)+p}=\left(p^{*}\right)^{\prime} .
$$

Then the family $\left\{u_{n}\right\}$ is uniformly bounded in $W_{0}^{1, p}(\Omega)$.

If $p=N$ the same result follows assuming that $f \in L^{m}(\Omega)$ for some $m>1$ while in the case $p>N$ the result is true if $f \in L^{1}(\Omega)$. 
Proof. We prove the result in the case $1<p<N$. Putting $u_{n}$ as test function in the weak formulation of (3.2), we get

$$
\begin{aligned}
\int_{\Omega}\left|\nabla u_{n}\right|^{p} & =\int_{\Omega} f_{n} g_{h}\left(u_{n}\right) u_{n} \leq h \int_{\Omega} f_{n} u_{n} \leq h \int_{\Omega} f u_{n} \\
& \leq h\left(\int_{\Omega} f^{m}\right)^{\frac{1}{m}}\left(\int_{\Omega} u_{n}^{p^{*}}\right)^{\frac{1}{p^{*}}} \leq C h\|f\|_{L^{m}(\Omega)}\left(\int_{\Omega}\left|\nabla u_{n}\right|^{p}\right)^{\frac{1}{p}},
\end{aligned}
$$

so that

$$
\left\|\nabla u_{n}\right\|_{L^{p}(\Omega)} \leq\left(C h\|f\|_{L^{m}(\Omega)}\right)^{\frac{1}{p-1}}
$$

which gives the uniform boundedness of $\left(u_{n}\right)$ in $W_{0}^{1, p}(\Omega)$.

Theorem 3.5. Let $1<p<N$ and $f \in L^{m}(\Omega)$, with

$$
m=\frac{N p}{N(p-1)+p}=\left(p^{*}\right)^{\prime} .
$$

Then (3.1) has a solution $u \in W_{0}^{1, p}(\Omega)$.

If $p=N$ the same result follows assuming that $f \in L^{m}(\Omega)$ for some $m>1$ while in the case $p>N$ the result is true if $f \in L^{1}(\Omega)$.

Proof. Let $u_{n}$ be the solution to (3.2) found in Lemma 3.1. By Lemma 3.4 we know that $\left\{u_{n}\right\}$ is uniformly bounded in $W_{0}^{1, p}(\Omega)$. It follows, up to subsequences, that $u_{n} \stackrel{W_{0}^{1, p}(\Omega)}{\rightarrow} u, u_{n} \stackrel{L^{p}(\Omega)}{\rightarrow} u$ and $u_{n}(x) \rightarrow u(x)$ a.e. in $\Omega$.

We will show that that $u$ is a solution to (3.1) proving the theorem. To prove this let $\varphi \in C_{c}^{\infty}(\Omega)$. Since by Lemma 3.3 we know that $u_{n}$ is bounded away from zero in the interior of the domain, we can exploit the results in $[2,9]$ to deduce that $\nabla u_{n}(x) \rightarrow \nabla u(x)$ a.e. in $\Omega$ which implies

$$
\left(\left|\nabla u_{n}(x)\right|+|\nabla u(x)|\right)^{p-2} \nabla\left(u_{n}(x)-u(x)\right) \rightarrow 0 \quad \text { a.e. in } \Omega .
$$

By Holder's inequality, we get

$$
\begin{aligned}
& \int_{\Omega}\left(\left|\nabla u_{n}\right|+|\nabla u|\right)^{p-2}\left|\nabla\left(u_{n}-u\right)\right||\nabla \varphi| \\
& \quad \leq \int_{\Omega}\left(\left|\nabla u_{n}\right|+|\nabla u|\right)^{p-1}|\nabla \varphi| \leq\|\nabla \varphi\|_{L^{\infty}(\Omega)}\left(\int_{\Omega}\left(\left|\nabla u_{n}\right|+|\nabla u|\right)^{p}\right)^{\frac{p-1}{p}}|\Omega|^{\frac{1}{p}} \\
& \quad \leq C\|\nabla \varphi\|_{L^{\infty}(\Omega)}\left(\left\|\nabla u_{n}\right\|_{L^{p}(\Omega)}+\|\nabla u\|_{L^{p}(\Omega)}\right)^{p-1}|\Omega|^{\frac{1}{p}} \leq C|\Omega|^{\frac{1}{p}}
\end{aligned}
$$

Therefore, exploiting Vitali's Theorem, we get that

$$
\begin{aligned}
& \left|\int_{\Omega}\left(\left|\nabla u_{n}\right|^{p-2} \nabla u_{n}-|\nabla u|^{p-2} \nabla u\right) \nabla \varphi\right| \\
& \quad \leq C \int_{\Omega}\left(\left|\nabla u_{n}\right|+|\nabla u|\right)^{p-2}\left|\nabla\left(u_{n}-u\right)\right||\nabla \varphi| \rightarrow 0,
\end{aligned}
$$

and

$$
\int_{\Omega}\left|\nabla u_{n}\right|^{p-2} \nabla u_{n} \nabla \varphi \rightarrow \int_{\Omega}|\nabla u|^{p-2} \nabla u \nabla \varphi
$$


It is easy to pass to the limit in right hand side of (3.2) and get that

$$
\int_{\Omega}|\nabla u|^{p-2} \nabla u \nabla \varphi=\int_{\Omega} f g_{h}(u) \varphi
$$

proving the result.

In the following, using the solution found in Theorem 3.5, we are going to prove some comparison principles that shall allow to obtain the uniqueness result. The case $\gamma<1, \gamma=1$ and $\gamma>1$ exhibits some technical differences and we will split the results in separate lemmata.

Lemma 3.6. Let $1<p<N$ and $f \in L^{m}(\Omega)$, with

$$
m=\frac{N p}{N(p-1)+p}=\left(p^{*}\right)^{\prime} .
$$

Moreover, let $u \in W_{\text {loc }}^{1, p}(\Omega)$ be a subsolution to (1.1) and let $w$ be the solution to (3.1). Then

$$
u \leq w+h^{-\frac{1}{\gamma}}
$$

with $h>0$ as in (3.1).

If $p=N$ the same result follows assuming that $f \in L^{m}(\Omega)$ for some $m>1$ while in the case $p>N$ the result is true if $f \in L^{1}(\Omega)$.

Proof. We fix $\varepsilon>0$ such that

$$
\frac{1}{\varepsilon^{\gamma}}<h
$$

Exploiting the fact that $w \in W_{0}^{1, p}(\Omega)$ it follows that

$$
(u-w-\varepsilon)^{+} \in W_{0}^{1, p}(\Omega) .
$$

Therefore, setting $T_{\tau}(s)=\min \{s, \tau\}$ for $s \geq 0$, by density arguments we can put $T_{\tau}\left((u-w-\varepsilon)^{+}\right)$as test function in (3.1) and get

$$
\int_{\Omega}|\nabla w|^{p-2} \nabla w \nabla T_{\tau}\left((u-w-\varepsilon)^{+}\right)=\int_{\Omega} f g_{h}(w) T_{\tau}\left((u-w-\varepsilon)^{+}\right) .
$$

Let now $\varphi_{n} \in C_{c}^{\infty}(\Omega)$ with

$$
\varphi_{n} \stackrel{W_{0}^{1, p}(\Omega)}{\rightarrow}(u-w-\varepsilon)^{+}
$$

and set

$$
\widetilde{\varphi}_{n, \tau}=T_{\tau}\left(\min \left\{(u-w-\varepsilon)^{+}, \varphi_{n}^{+}\right\}\right) .
$$

Then $\widetilde{\varphi}_{n, \tau} \in W_{0}^{1, p}(\Omega) \cap L_{c}^{\infty}(\Omega)$ and consequently:

$$
\int_{\Omega}|\nabla u|^{p-2} \nabla u \nabla \widetilde{\varphi}_{n, \tau} \leq \int_{\Omega} \frac{f}{u^{\gamma}} \widetilde{\varphi}_{n, \tau} .
$$

Since $|\nabla u|^{p}$ is integrable in the support of $(u-w-\varepsilon)^{+}$(see Definitiom 1.2), we can pass to the limit and deduce that

$$
\int_{\Omega}|\nabla u|^{p-2} \nabla u \nabla T_{\tau}\left((u-w-\varepsilon)^{+}\right) \leq \int_{\Omega} \frac{f}{u^{\gamma}} T_{\tau}\left((u-w-\varepsilon)^{+}\right) .
$$


By (3.4) and (3.5) it follows:

$$
\begin{aligned}
& C \int_{\Omega}(|\nabla w|+|\nabla u|)^{p-2}\left|\nabla T_{\tau}\left((u-w-\varepsilon)^{+}\right)\right|^{2} \\
& \quad \leq \int_{\Omega}\left(|\nabla u|^{p-2} \nabla u-|\nabla w|^{p-2} \nabla w\right) \nabla T_{\tau}\left((u-w-\varepsilon)^{+}\right) \\
& \quad \leq \int_{\Omega} \frac{f}{u^{\gamma}} T_{\tau}\left((u-w-\varepsilon)^{+}\right)-\int_{\Omega} f g_{h}(w) T_{\tau}\left((u-w-\varepsilon)^{+}\right) \\
& \quad \leq \int_{\Omega} f\left(\frac{1}{u^{\gamma}}-g_{h}(w)\right) T_{\tau}\left((u-w-\varepsilon)^{+}\right) \\
& \quad=\int_{\Omega} f\left(g_{h}(u)-g_{h}(w)\right) T_{\tau}\left((u-w-\varepsilon)^{+}\right) \\
& \leq 0
\end{aligned}
$$

where the last inequality is obtained exploiting the fact that $\varepsilon>h^{-\frac{1}{\gamma}}$ (and recalling the definition of $g_{h}$ ) and exploiting the fact that $u \geq \varepsilon$ in the support of $(u-w-\varepsilon)^{+}$. Therefore we have

$$
\int_{\Omega}(|\nabla u|+|\nabla w|)^{p-2}\left|\nabla T_{\tau}\left((u-w-\varepsilon)^{+}\right)\right|^{2}=0,
$$

which implies $T_{\tau}\left((u-w-\varepsilon)^{+}\right)=0$ a.e. in $\Omega$. By the arbitrariness of $\tau>0$, we obtain $u-w-\varepsilon \leq 0$ and the thesis is proved.

Lemma 3.7. Let $1<p<N$ and $f \in L^{m}(\Omega)$, with $m>\frac{N}{p}$. Moreover, let $v \in W_{l o c}^{1, p}(\Omega)$ be a supersolution of (1.1) and let $w$ be the solution to (3.1).

Then $w \leq v$.

If $p=N$ the same result follows assuming that $f \in L^{m}(\Omega)$ for some $m>1$ while in the case $p>N$ the result is true if $f \in L^{1}(\Omega)$.

Proof. We consider the case $1<p<N$. Since the regularized equation in (3.1) is not singular, by the assumption $m>\frac{N}{p}$ and standard regularity theory, it follows that $w$ is Holder continuous up to the boundary.

Since $w=0$ on $\partial \Omega$, it follows that there exists $\delta>0$ such that $w \leq \frac{\varepsilon}{2}$ in $A_{\delta}=\{x \in \bar{\Omega}: \operatorname{dist}(x, \partial \Omega)<\delta\}$. Then $w-v-\varepsilon \leq-\frac{\varepsilon}{2}<0$ in $A_{\delta}$ so that the support of $(w-v-\varepsilon)^{+}$is contained in $\Omega \backslash A_{\delta} \subset \subset \Omega$. Since $(w-v-\varepsilon)^{+} \in W_{0}^{1, p}(\Omega)$ we can therefore argue as in Lemma 3.6 and get

$$
\int_{\Omega}|\nabla w|^{p-2} \nabla w \nabla T_{\tau}\left((w-v-\varepsilon)^{+}\right)=\int_{\Omega} f g_{h}(w) T_{\tau}\left((w-v-\varepsilon)^{+}\right),
$$

where $T_{\tau}(s)=\min \{s, \tau\}$ for $s \geq 0$. Let now $\varphi_{n} \in C_{c}^{\infty}(\Omega)$ with

$$
\varphi_{n} \stackrel{W_{0}^{1, p}(\Omega)}{\rightarrow}(w-v-\varepsilon)^{+} ; \quad \widetilde{\varphi}_{n, \tau}=T_{\tau}\left(\min \left\{(w-v-\varepsilon)^{+}, \varphi_{n}^{+}\right\}\right) .
$$

Then $\widetilde{\varphi}_{n, \tau} \in W_{0}^{1, p}(\Omega) \cap L_{c}^{\infty}(\Omega)$ and

$$
\int_{\Omega}|\nabla v|^{p-2} \nabla v \nabla \widetilde{\varphi}_{n, \tau} \geq \int_{\Omega} \frac{f}{v^{\gamma}} \widetilde{\varphi}_{n, \tau}
$$


and, passing to the limit, we deduce that

$$
\int_{\Omega}|\nabla v|^{p-2} \nabla v \nabla T_{\tau}\left((w-v-\varepsilon)^{+}\right) \geq \int_{\Omega} \frac{f}{v^{\gamma}} T_{\tau}\left((w-v-\varepsilon)^{+}\right) .
$$

To prove this it is crucial the fact that the support of $(w-v-\varepsilon)^{+}$is contained in $\Omega \backslash A_{\delta} \subset \subset \Omega$ since we only know that $v \in W_{\text {loc }}^{1, p}(\Omega)$. By (3.6) and (3.7) now we get

$$
\begin{aligned}
& C \int_{\Omega}(|\nabla w|+|\nabla v|)^{p-2}\left|\nabla T_{\tau}\left((w-v-\varepsilon)^{+}\right)\right|^{2} \\
& \quad \leq \int_{\Omega}\left(|\nabla w|^{p-2} \nabla w-|\nabla v|^{p-2} \nabla v\right) \nabla T_{\tau}\left((w-v-\varepsilon)^{+}\right) \\
& \quad \leq \int_{\Omega} f g_{h}(w) T_{\tau}\left((w-v-\varepsilon)^{+}\right)-\int_{\Omega} \frac{f}{v^{\gamma}} T_{\tau}\left((w-v-\varepsilon)^{+}\right) \\
& \quad \leq \int_{\Omega} f\left(g_{h}(w)-\frac{1}{v^{\gamma}}\right) T_{\tau}\left((w-v-\varepsilon)^{+}\right) \\
& \quad \leq \int_{\Omega} f\left(\frac{1}{w^{\gamma}}-\frac{1}{v^{\gamma}}\right) T_{\tau}\left((w-v-\varepsilon)^{+}\right) \leq 0
\end{aligned}
$$

so that

$$
\int_{\Omega}(|\nabla w|+|\nabla v|)^{p-2}\left|\nabla T_{\tau}\left((w-v-\varepsilon)^{+}\right)\right|^{2}=0,
$$

which implies $T_{\tau}\left((w-v-\varepsilon)^{+}\right)=0$ a.e. in $\Omega$. By the arbitrariness of $\tau>0$, we obtain that $w-v-\varepsilon \leq 0$ and, passing to the limit as $\varepsilon \rightarrow 0$, it follows that $w \leq v$.

Remark 3.8. The reader should note that in Lemma 3.6 we do not pass to the limit because $w$ depends on $h$.

We are now ready to prove Theorem 1.5 in the case $\gamma>1$.

Proof of Theorem 1.5, the case $\gamma>1$. Let $u$ and $v$ be solutions of (1.1) and let $w$ be the solution to (3.1). By Lemma 3.6 and Lemma 3.7, we get $u \leq w+h^{-\frac{1}{\gamma}}$ and $w \leq v$ so that, since $h$ is arbitrary, we get that $u \leq v$. In the same way it follows that $v \leq u$ which implies $u=v$ and the thesis is proved.

The uniqueness result in the case $0<\gamma \leq 1$ is simpler, mainly because of the fact that solutions are in $W_{0}^{1, p}(\Omega)$ in this case. We provide a short proof here below.

Proof of Theorem 1.5, the case $0<\gamma \leq 1$. Let $u, v \in W_{0}^{1, p}(\Omega)$ be solutions to $(1.1)$. Since $(u-v)^{+} \in W_{0}^{1, p}(\Omega)$ we can take a sequence $\varphi_{n} \in C_{c}^{\infty}(\Omega)$

$$
\varphi_{n} \stackrel{W_{0}^{1, p}(\Omega)}{\rightarrow}(u-v)^{+} .
$$

Set

$$
\widetilde{\varphi}_{n}=\min \left\{(u-v)^{+}, \varphi_{n}^{+}\right\} \in W_{0}^{1, p}(\Omega) \cap L_{c}^{\infty}
$$


so that

$$
\begin{gathered}
\int_{\Omega}\left(|\nabla u|^{p-2} \nabla u-|\nabla v|^{p-2} \nabla v\right) \nabla \widetilde{\varphi}_{n} \\
\leq \int_{\Omega} f\left(\frac{1}{u^{\gamma}}-\frac{1}{v^{\gamma}}\right) \widetilde{\varphi}_{n} \leq 0 .
\end{gathered}
$$

Passing to the limit we obtain

$$
\int_{\Omega}(|\nabla u|+|\nabla v|)^{p-2}\left|\nabla(u-v)^{+}\right|^{2}=0,
$$

and $(u-v)^{+}=0$ a.e. in $\Omega$. It follows $u \leq v$ and the thesis is proved reversing the roles of $u$ and $v$.

\section{Appendix: Existence of the solution}

We start considering the following problem

$$
\left\{\begin{array}{cl}
-\Delta_{p} u_{n}=\frac{f_{n}(x)}{\left(u_{n}+\frac{1}{n}\right)^{\gamma}} & \text { in } \Omega, \\
u_{n}=0 & \text { on } \partial \Omega,
\end{array}\right.
$$

with $f_{n}=\min \{f, n\}$. Arguing exactly as in Lemmas 3.1 and 3.3, one can easily prove the following:

Lemma 4.1. For each $n \in \mathbb{N}$ problem (4.1) has a solution $u_{n} \in W_{0}^{1, p}(\Omega) \cap$ $L^{\infty}(\Omega)$ and $u_{n}>0$ in $\Omega$. The sequence $\left\{u_{n}\right\}$ is increasing with respect to $n$ and

$$
\forall \omega \subset \subset \Omega \exists c_{\omega}: \forall n \in \mathbb{N}, u_{n} \geq c_{\omega}>0 \quad \text { in } \omega .
$$

From this we immediately deduce the following:

Theorem 4.2. If $\gamma=1$ and $f \in L^{1}(\Omega)$ then (1.1) has a solution $u \in W_{0}^{1, p}(\Omega)$.

Proof. It is standard to prove that the sequence $\left\{u_{n}\right\}$ given by Lemma 4.1 is uniformly bounded in $W_{0}^{1, p}(\Omega)$. Therefore we can obtain a solution passing to the limit, namely arguing exactly as in Theorem 3.5.

Lemma 4.3. Let $1<p<N, \gamma>1$ and let $u_{n}$ be the solution to (4.1). Assume that $f \in L^{1}(\Omega)$. Then $\left\{u_{n}^{\frac{\gamma+p-1}{p}}\right\}$ is uniformly bounded in $W_{0}^{1, p}(\Omega),\left\{u_{n}\right\}$ is uniformly bounded in $W_{l o c}^{1, p}(\Omega)$ and in $L^{s}(\Omega)$, with

$$
s=\frac{N(\gamma+p-1)}{N-p}=\frac{p^{*}(\gamma+p-1)}{p} .
$$

In the case $p \geq N$ the result holds and (4.2) holds for any $s \geq 1$.

Proof. We consider the case $1<p<N$. The changes needed in the case $p \geq N$ are trivial. Let us insert $u_{n}^{\gamma}$ as test function in (4.1) obtaining

$$
\gamma \int_{\Omega}\left|\nabla u_{n}\right|^{p} u_{n}^{\gamma-1}=\int_{\Omega} \frac{f_{n} u_{n}^{\gamma}}{\left(u_{n}+\frac{1}{n}\right)^{\gamma}} \leq \int_{\Omega} f_{n} \leq \int_{\Omega} f=\|f\|_{L^{1}(\Omega)} .
$$


Therefore

$$
\left\|\nabla\left(u_{n}^{\frac{\gamma+p-1}{p}}\right)\right\|_{L^{p}(\Omega)} \leq C\|f\|_{L^{1}(\Omega)}^{\frac{1}{p}}
$$

and the first part of the thesis is proved. Recalling the assumption on the exponent $s$ we deduce that

$$
\begin{aligned}
\left\|u_{n}\right\|_{L^{s}(\Omega)} & =\left(\int_{\Omega} u_{n}^{\frac{p^{*}(\gamma+p-1)}{p}}\right)^{\frac{1}{s}}=\left\|u_{n}^{\frac{\gamma+p-1}{p}}\right\|_{L^{p^{*}}(\Omega)}^{\frac{p^{*}}{s}} \\
& \leq C\left\|\nabla\left(u_{n}^{\frac{\gamma+p-1}{p}}\right)\right\|_{L^{p}(\Omega)}^{\frac{p^{*}}{s}} \leq C\|f\|_{L^{1}(\Omega)}^{\frac{p^{*}}{p s}},
\end{aligned}
$$

which gives the uniform boundedness of $\left\{u_{n}\right\}$ in $L^{s}(\Omega)$.

To prove the uniform boundedness of $\left\{u_{n}\right\}$ in $W_{l o c}^{1, p}(\Omega)$ it could be enough to use $u_{n} \varphi^{2}$ as test function in (4.1). Anyway this is also a simple consequence of the first part of the theorem, namely the fact that $\left\{u_{n}^{\frac{\gamma+p-1}{p}}\right\}$ is uniformly bounded in $W_{0}^{1, p}(\Omega)$ and the fact that $\left\{u_{n}\right\}$ is bounded away from zero on compact sets.

Theorem 4.4. Let $\gamma>1$ and let $f \in L^{1}(\Omega)$. Then (1.1) has a solution $u \in$ $W_{\text {loc }}^{1, p}(\Omega)$. Moreover $u^{\frac{\gamma+p-1}{p}} \in W_{0}^{1, p}(\Omega)$.

Proof. By Lemma 4.3 we have that the sequence $\left\{u_{n}\right\}$ of solutions to the regularized problem (4.1) is uniformly bounded in $W_{l o c}^{1, p}(\Omega)$.

It follows, up to subsequences, that $u_{n} \stackrel{W_{\text {loc }}^{1, p}(\Omega)}{\longrightarrow} u, u_{n} \stackrel{L_{\text {loc }}^{p}(\Omega)}{\rightarrow} u$ and $u_{n}(x)$ $\rightarrow u(x)$ a.e. in $\Omega$. Let $\varphi \in C_{c}^{\infty}(\Omega)$ and let $\omega$ be the support of $\varphi$. Recalling that the sequence $u_{n}$ is bounded away from zero in the interior of the domain, we can apply $[2,9]$ to show that $\nabla u_{n}(x) \rightarrow \nabla u(x)$ a.e. in $\Omega$ which implies

$$
\left(\left|\nabla u_{n}(x)\right|+|\nabla u(x)|\right)^{p-2} \nabla\left(u_{n}(x)-u(x)\right) \rightarrow 0 \quad \text { a. e. in } \Omega .
$$

Furthermore, by Holder inequality, we get

$$
\begin{aligned}
& \int_{\Omega}\left(\left|\nabla u_{n}\right|+|\nabla u|\right)^{p-2}\left|\nabla\left(u_{n}-u\right)\right||\nabla \varphi| \\
& \quad \leq \int_{\Omega}\left(\left|\nabla u_{n}\right|+|\nabla u|\right)^{p-1}|\nabla \varphi| \leq\|\nabla \varphi\|_{L^{\infty}(\Omega)}\left(\int_{\omega}\left(\left|\nabla u_{n}\right|+|\nabla u|\right)^{p}\right)^{\frac{p-1}{p}}|\Omega|^{\frac{1}{p}} \\
& \quad \leq C(\omega)|\Omega|^{\frac{1}{p}} .
\end{aligned}
$$

Therefore we can exploit Vitali's Theorem to get

$$
\begin{aligned}
& \left|\int_{\Omega}\left(\left|\nabla u_{n}\right|^{p-2} \nabla u_{n}-|\nabla u|^{p-2} \nabla u\right) \nabla \varphi\right| \\
& \quad \leq C \int_{\Omega}\left(\left|\nabla u_{n}\right|+|\nabla u|\right)^{p-2}\left|\nabla\left(u_{n}-u\right)\right||\nabla \varphi| \rightarrow 0,
\end{aligned}
$$

so that 


$$
\int_{\Omega}\left|\nabla u_{n}\right|^{p-2} \nabla u_{n} \nabla \varphi \rightarrow \int_{\Omega}|\nabla u|^{p-2} \nabla u \nabla \varphi .
$$

Moreover, recalling Lemma 4.1, we have that

$$
\left|\frac{f_{n} \varphi}{\left(u_{n}+\frac{1}{n}\right)^{\gamma}}\right| \leq \frac{\|\varphi\|_{L^{\infty}(\Omega)}}{c_{\omega}^{\gamma}} f \text { in } \omega
$$

which allows to pass to the limit in (4.1) obtaining

$$
\int_{\Omega}|\nabla u|^{p-2} \nabla u \nabla \varphi=\int_{\Omega} \frac{f \varphi}{u^{\gamma}}
$$

showing that $u$ is a solution to (1.1). The fact that $u^{\frac{\gamma+p-1}{p}} \in W_{0}^{1, p}(\Omega)$ follows by Lemma 4.3 .

In the following we consider the case $0<\gamma<1$.

Lemma 4.5. Let $1<p<N, 0<\gamma<1$ and let $u_{n}$ be the solution to (4.1). Assume that $f \in L^{m}(\Omega)$ with

$$
m=\frac{N p}{N(p-1)+p+\gamma(N-p)}=\left(\frac{p^{*}}{1-\gamma}\right)^{\prime},
$$

then $\left\{u_{n}\right\}$ is uniformly bounded in $W_{0}^{1, p}(\Omega)$.

If $p=N$ the same result follows assuming that $f \in L^{m}(\Omega)$ for some $m>1$ while in the case $p>N$ the result is true if $f \in L^{1}(\Omega)$.

Proof. We only consider the case $1<p<N$. The other cases are simpler and can be proved in a similar way with trivial changes. We have that

$$
\int_{\Omega}\left|\nabla u_{n}\right|^{p}=\int_{\Omega} \frac{f_{n} u_{n}}{\left(u_{n}+\frac{1}{n}\right)^{\gamma}} \leq \int_{\Omega} f_{n} u_{n}^{1-\gamma} \leq\|f\|_{L^{m}(\Omega)}\left(\int_{\Omega} u_{n}^{(1-\gamma) m^{\prime}}\right)^{\frac{1}{m^{\prime}}}
$$

Since $(1-\gamma) m^{\prime}=p^{*}$, then by Sobolev inequality, we get

$$
\left(\int_{\Omega}\left|u_{n}\right|^{p^{*}}\right)^{\frac{p}{p^{*}}} \leq C \int_{\Omega}\left|\nabla u_{n}\right|^{p} \leq C\|f\|_{L^{m}(\Omega)}\left(\int_{\Omega} u_{n}^{p^{*}}\right)^{\frac{1}{m^{\prime}}} .
$$

Since $\frac{p}{p^{*}}>\frac{1}{m^{\prime}}$, by $(4.4)$, we get that

$$
\left\|u_{n}\right\|_{L^{p^{*}}(\Omega)} \leq\left(C\|f\|_{L^{m}(\Omega)}\right)^{\frac{1}{p^{*}\left(\frac{p}{p^{*}}-\frac{1}{m^{\prime}}\right)}} .
$$

From this, exploiting (4.3), we deduce that $\left\{u_{n}\right\}$ is uniformly bounded in $W_{0}^{1, p}(\Omega)$.

Theorem 4.6. Let $1<p<N$ and let $0<\gamma<1$ and assume that $f \in L^{m}(\Omega)$ with

$$
m=\frac{N p}{N(p-1)+p+\gamma(N-p)}=\left(\frac{p^{*}}{1-\gamma}\right)^{\prime} .
$$

Then (1.1) has a solution $u \in W_{0}^{1, p}(\Omega)$.

If $p=N$ the same result follows assuming that $f \in L^{m}(\Omega)$ for some $m>1$ while in the case $p>N$ the result is true if $f \in L^{1}(\Omega)$. 
Proof. The proof follows now exploiting Lemma 4.5 and passing to the limit arguing as in Theorems 4.2 and 4.4 .

Proof of Theorem 1.3. The proof of Theorem 1.3 follows collecting Theorems $4.2,4.4$ and 4.6 .

\section{References}

[1] Arcoya, D., Boccardo, L., Leonori, T., Porretta, A.: Some elliptic problems with singular natural growth lower order terms. J. Differ. Equ. 249(11), 27712795 (2010)

[2] Boccardo, L., Murat, F.: Almost everywhere convergence of the gradients of solutions to elliptic and parabolic equations. Nonlinear Anal. 19, 405-412 (1992)

[3] Brandolini, B., Chiacchio, F., Trombetti, C.: Symmetrization for singular semilinear elliptic equations. Ann. Mat. Pura Appl. 4(2), 389-404 (2014)

[4] Boccardo, L., Orsina, L.: Semilinear elliptic equations with singular nonlinearities. Calc. Var. Partial Differ. Equ. 37(3-4), 363-380 (2010)

[5] Canino, A., Degiovanni, M.: A variational approach to a class of singular semilinear elliptic equations. J. Convex Anal. 11(1), 147-162 (2004)

[6] Canino, A., Grandinetti, M., Sciunzi, B.: Symmetry of solutions of some semilinear elliptic equations with singular nonlinearities. J. Differ. Equ. 255(12), 44374447 (2013)

[7] Canino, A., Sciunzi, B.: A uniqueness result for some singular semilinear elliptic equations. Preprint available at: arXiv:1407.5984

[8] Crandall, M.G., Rabinowitz, P.H., Tartar, L.: On a Dirichlet problem with a singular nonlinearity. Commun. Partial Differ. Equ. 2(2), 193-222 (1977)

[9] Dal Maso, G., Murat, F.: Almost everywhere convergence of gradients of solutions to nonlinear elliptic systems. Nonlinear Anal. 31(3-4), 6, 581-597 (1998)

[10] Damascelli, L., Sciunzi, B.: Regularity, monotonicity and symmetry of positive solutions of $m$-Laplace equations. J. Differ. Equ. 206(2), 483-515 (2004)

[11] Damascelli, L., Sciunzi, B.: Harnack inequalities, maximum and comparison principles, and regularity of positive solutions of $m$-Laplace equations. Calc. Var. Partial Differ. Equ. 25(2), 139-159 (2006)

[12] Di Benedetto, E.: $C^{1+\alpha}$ local regularity of weak solutions of degenerate elliptic equations. Nonlinear Anal. 7(8), 827-850 (1983)

[13] Gatica, J.A., Oliker, V., Waltman, P.: Singular nonlinear boundary value problems for second-order ordinary differential equations. J. Differ. Equ. 79(1), 62$78(1989)$

[14] Giachetti, D., Murat, F.: An elliptic problem with a lower order term having singular behaviour. Boll. Unione Mat. Ital. (9) 2, 9(2), 349-370 (2009) 
[15] Hirano, N., Saccon, C., Shioji, N.: Multiple existence of positive solutions for singular elliptic problems with concave ad convex nonlinearities. Adv. Differ. Equ. 9(1-2), 197-220 (2004)

[16] Kawohl, B.: On a class of singular elliptic equations, in Progress in partial differential equations: elliptic and parabolic problems (Pont-à-Mousson, 1991) Pitman Res. Notes Math. Ser., vol. 266, Longman Sci. Tech. Harlow, pp. 156-163 (1992)

[17] Kuusi, T., Mingione, G.: Universal potential estimates. J. Funct. Anal. 262, 4205-4269 (2012)

[18] Lair, A.V., Shaker, A.W.: Classical and weak solutionsof a singular semilinear elliptic problem. J. Math. Anal. Appl. 211(2), 371-385 (1977)

[19] Lazer, A.C., McKenna, P.J.: On a singular nonlinear elliptic boundary-value problem. Proc. Am. Math. Soc. 111, 721-730 (1991)

[20] Lieberman, G.M.: Boundary regularity for solutions of degenerate elliptic equations. Nonlinear Anal. 12(11), 1203-1219 (1988)

[21] Pucci, P., Serrin, J.: The Maximum Principle. Birkhauser, Boston (2007)

[22] Stuart, C.A.: Existence and approximation of solutions of non-linear elliptic equations. Math. Z. 147, 53-63 (1976)

[23] Sciunzi, B.: Some results on the qualitative properties of positive solutions of quasilinear elliptic equations. NoDEA. Nonlinear Differ. Equ. Appl. 14(34), 315-334 (2007)

[24] Sciunzi, B.: Regularity and comparison principles for p-Laplace equations with vanishing source term. Commun. Contemp. Math. 16(6), 1450013, 20 (2014)

[25] Teixeira, E.: Regularity for quasilinear equations on degenerate singular sets. Math. Ann. 358(1-2), 241-256 (2014)

[26] Tolksdorf, P.: Regularity for a more general class of quasilinear elliptic equations. J. Differ. Equ. 51(1), 126-150 (1984)

[27] Vázquez, J.L.: A strong maximum principle for some quasilinear elliptic equations. Appl. Math. Optim. 12(3), 191-202 (1984)

Annamaria Canino, Berardino Sciunzi and Alessandro Trombetta

Dipartimento di Matematica e Informatica

Università della Calabria

Ponte Pietro Bucci 31B

87036 Arcavacata di Rende, Cosenza

Italy

e-mail: canino@mat.unical.it

sciunzi@mat.unical.it

alessandro.trombetta@unical.it

Received: 1 April 2015.

Accepted: 17 October 2015. 